\title{
Developing the wide-spectral coverage, very high-efficiency grisms for MOIRCS on Subaru Telescope (Erratum)
}

Ichi Tanaka, Noboru Ebizuka, Kentaro Motohara, Tadayuki Kodama, Takashi Hattori, et al.

Ichi Tanaka, Noboru Ebizuka, Kentaro Motohara, Tadayuki Kodama, Takashi Hattori, Koji Omata, Hidenori Takahashi, Masahiro Konishi, Yoko Tanaka, "Developing the wide-spectral coverage, very high-efficiency grisms for MOIRCS on Subaru Telescope (Erratum)," Proc. SPIE 11451, Advances in Optical and Mechanical Technologies for Telescopes and Instrumentation IV, 1145174 (18 May 2021); doi: 10.1117/12.2601442 


\section{Developing the wide-spectral coverage, very high- efficiency grisms for MOIRCS on Subaru Telescope (Erratum)}

Ichi Tanaka, Subaru Telescope, Noboru Ebizuka, Riken, Kentaro Motohara, The Univ. of Tokyo, Takashi Hattori, Subaru Telescope, Koji Omata, Subaru Telescope, Hidenori Takahashi, The Univ. of Tokyo, Masahiro Konishi, The Univ. of Tokyo and Yoko Tanaka, Subaru Telescope

Proceedings Volume 11451, Advances in Optical and Mechanical Technologies for Telescopes and Instrumentation IV; $114515 \mathrm{~A}$ (2020) https://doi.org/10.1117/12.2561176

Event: Advances in Optical and Mechanical Technologies for Telescopes and Instrumentation IV

Online Publication Date: 13 December 2020

Erratum Published: 18 May 2021

A revised version of this manuscript was published on 18 May 2021. Details of the revision are provided in the text that accompanies this Erratum. The original paper has been updated. 